\title{
Coarse graining the state space of a turbulent flow using periodic orbits
}

\author{
Gökhan Yalnız, ${ }^{1,2}$ Björn Hof, ${ }^{1}$ and Nazmi Burak Budanur ${ }^{1}$ \\ ${ }^{1}$ IST Austria, 3400 Klosterneuburg, Austria \\ ${ }^{2}$ Physics Department, Boğaziçi University, 34342 Istanbul, Turkey
}

(Dated: April 29, 2021)

\begin{abstract}
We show that turbulent dynamics that arise in simulations of the three-dimensional Navier-Stokes equations in a triply-periodic domain under sinusoidal forcing can be described as transient visits to the neighborhoods of unstable time-periodic solutions. Based on this description, we reduce the original system with more than $10^{5}$ degrees of freedom to a 17-node Markov chain where each node corresponds to the neighborhood of a periodic orbit. The model accurately reproduces long-term averages of the system's observables as weighted sums over the periodic orbits.
\end{abstract}

Producing low-dimensional models of turbulent flows has been a long-standing scientific challenge with a wide potential for applications. Following the discoveries [1-6] of unstable time-invariant solutions (equilibria, traveling waves, $\ldots)$ of three-dimensional $(3 D)$ fluid flows in pipes and channels, Gibson et al. [7] demonstrated the influence of invariant solutions on the dynamics of plane Couette flow through state space visualizations. Although the underlying hypothesis that such solutions could eventually be used for turbulence modeling has been discussed in subsequent studies [8-11], a clear path towards this goal remained missing.

The studies of invariant solutions of turbulent flows are founded upon a view of fluid dynamics as a highdimensional dynamical system [12]. In a computational setting, such a dynamical system is constructed by a spatial discretization that yields a numerical representation of the fluid's state and a simulator that sets the time-evolution rule. The simplest invariant solutions of continuous-time dynamical systems are equilibria, which in fluid dynamics correspond to velocity fields that are stationary. Even though equilibria can influence chaotic flows through their stable and unstable manifolds [7, 13], they by definition lack dynamics and on their own cannot be used for modeling. At the focus of the present work are periodic orbits (POs), which form loops in the state space and correspond to velocity fields that recur exactly after a constant period.

Unstable POs that are embedded in strange attractors offer a systematic way of exploring chaos since the POs and the chaotic trajectories in their vicinity have similar physical properties [14]. However, the instability of POs necessitates special methods for their numerical discovery and poses a technical challenge especially in high-dimensional settings such as shear flow turbulence. Extensive searches for POs in high-dimensional systems have become possible after Viswanath's introduction of the Newton-Krylov-hookstep algorithm [15]. Since then, many POs were computed in plane Couette [8] and pipe [10] flows where similarities between turbulence and POs were observed. However, in these studies no attempt was made to construct a turbulence model based on POs.

In this Letter, we present a quantitatively accurate reduced-order model of a $3 D$ shear flow based on the numerically computed periodic solutions of the governing equations. Specifically, we consider $3 D$ Kolmogorov flow [16] under certain symmetry restrictions and utilize the recently-introduced [17] state space persistence analysis for quantifying similarities between turbulence and POs to show that the dynamics of this system can be decomposed into consecutive visits to the neighborhoods of the POs. Consequently, we propose the neighborhoods of POs as the bases of a Markov process that serves as a coarse-grained model of the turbulent flow. Upon comparing the long-term observable averages from simulations to those obtained from the invariant distribution of the Markov chain, we show that the POs give an approximation to the natural measure [18-20] of the system.

$3 D$ Kolmogorov flow is described by the body-forced Navier-Stokes equations

$$
\mathbf{u}_{t}+\mathbf{u} \cdot \nabla \mathbf{u}=-\nabla p+\nu \nabla^{2} \mathbf{u}+\mathbf{f}
$$

in a rectangular box $\left[0, L_{x}\right] \times\left[0, L_{y}\right] \times\left[0, L_{z}\right]$, where $\mathbf{u}=[u, v, w](x, y, z)$ and $p=p(x, y, z)$ are the velocity and pressure fields respectively, $\nu$ is the kinematic viscosity, $\mathbf{f}=\gamma \sin \left(2 \pi y / L_{y}\right) \hat{\mathbf{e}}_{x}$ is the body force with amplitude $\gamma$ and $\hat{\mathbf{e}}_{x}$ denotes the unit vector in the $x$ direction. $\mathbf{u}$ satisfies the incompressibility condition $\nabla \cdot \mathbf{u}=0$ and periodic boundary conditions in all three directions. The laminar solution of (1) is given by $u_{L}=\gamma \nu^{-1}\left(L_{y} /(2 \pi)\right)^{2} \sin \left(2 \pi y / L_{y}\right), v_{L}=0, w_{L}=0$ and it is linearly stable for all $\nu$ [21]. Nevertheless, turbulence can be triggered by finite-amplitude perturbations and is transient at high $\nu$ [21]. In this sense, $3 D$ Kolmogorov flow admits the basic phenomenology of the transitional turbulence in wall-bounded shear flows such as those in pipes and channels [22].

For numerical integration of (1), we developed dnsbox [23], a pseudospectral [24, 25] solver based on the hit3d code [26]. We adapted the Newton-Krylovhookstep implementation of Openpipeflow [27] for finding POs and utilized scikit-tda [28] for topological data 
analysis. In what follows, we set $\nu=0.05, \gamma=1.0$, and $L_{x} \times L_{y} \times L_{z}=2 \pi \times 2 \pi \times \pi$. The numbers of spatial grid points are $\left[N_{x}, N_{y}, N_{z}\right]=[64,64,32]$, and the second-order predictor-corrector time step is $\Delta t=$ 0.0025 . Fourier-expanded fields are dealiased following the $2 / 3$ rule and the Fourier coefficients show at least four orders of magnitude drop-off at all times in each direction [29]. The number of nonzero Fourier coefficients after dealiasing is 110946. This is an upper bound on the dimension of our system, which, in practice, is reduced by the divergence-free condition and the imposed symmetries. In the supplemental material (SM) [29] we provide estimates for the effective number of degrees of freedom.

3D Kolmogorov flow is equivariant under the continuous translations $T_{x}(\delta x)$ and $T_{z}(\delta z)$ in $x$ and $z$ directions by $\delta x$ and $\delta z$, respectively, and the discrete symmetries [21]

$$
\begin{aligned}
R_{x y}[u, v, w](x, y, z) & =[-u,-v, w](-x,-y, z) \\
R_{y}[u, v, w](x, y, z) & =[u,-v, w]\left(x,-y-L_{y} / 2, z\right) \\
R_{z}[u, v, w](x, y, z) & =[u, v,-w](x, y,-z) \\
S_{x}[u, v, w](x, y, z) & =[-u, v, w]\left(-x, y-L_{y} / 2, z\right)
\end{aligned}
$$

We restrict our study to the flow-invariant subspace of the velocity fields that are symmetric under $S_{x}$ and $R_{z}$, in which complications due to the continuous symmetries [30] are avoided since only the translations by $L_{x} / 2$ and $L_{z} / 2$ in $x$ and $z$ directions respectively are allowed. This flow-invariant subspace still exhibits transient turbulence with lifetimes of $O(1000)$, more than 300 times the period of our shortest PO, i.e. the shortest characteristic turnover time. Since invariance under $S_{x}$ equates the action of $R_{x y}$ and $R_{y}$, we can write the symmetry group of the system as

$$
\begin{aligned}
G= & \left\{I, T_{x / 2}, T_{z / 2}, R_{x y}, T_{x / 2} T_{z / 2}, T_{z / 2} R_{x y},\right. \\
& \left.T_{x / 2} R_{x y}, T_{x / 2} T_{z / 2} R_{x y}\right\}
\end{aligned}
$$

where $T_{x / 2}=T_{x}\left(L_{x} / 2\right), T_{z / 2}=T_{z}\left(L_{z} / 2\right)$, and $I$ is identity.

The presence of symmetries (6) implies that each generic state of the system has 7 symmetry copies. Since our analyses require parsing large data sets, it is crucial to eliminate redundancies in the data. With this in mind, we construct a symmetry-reduced representation of our system via a state space coordinate transformation. Let $\tilde{\xi}$ be a state vector holding the real and imaginary parts of coefficients in the Fourier expansion of $\mathbf{u}$. Noting that each element of (6) is its own inverse, we decompose $\tilde{\xi}$ into symmetric and antisymmetric components under the action of $\sigma \in G$ as $\tilde{\xi}_{\sigma}^{ \pm}=\frac{1}{\sqrt{2}}(I \pm \sigma) \tilde{\xi}$. By construction under the action of $\sigma$, the elements of $\tilde{\xi}_{\sigma}^{+}$are invariant and those of $\tilde{\xi}_{\sigma}^{-}$change signs. Let $\left(\rho_{1}, \rho_{2}, \rho_{3}, \rho_{4}, \ldots\right)$ be the elements of $\tilde{\xi}_{\sigma}^{-}$, we write the invariants of $\sigma$ as

$$
\left\{\frac{\rho_{1}^{2}-\rho_{2}^{2}}{\sqrt{\rho_{1}^{2}+\rho_{2}^{2}}}, \frac{\rho_{1} \rho_{2}}{\sqrt{\rho_{1}^{2}+\rho_{2}^{2}}}, \frac{\rho_{2} \rho_{3}}{\sqrt{\rho_{2}^{2}+\rho_{3}^{2}}}, \frac{\rho_{3} \rho_{4}}{\sqrt{\rho_{3}^{2}+\rho_{4}^{2}}}, \ldots\right\} .
$$

These invariants, without the denominators, were written for the Kuramoto-Sivashinsky system in Ref. [31]. Here, we introduce the denominators to prevent the transformation from producing numbers that are too large or small. One can confirm by inspection that the elements of (7) are invariant when all $\rho_{i}$ change their signs but not when any other subset of $\rho_{i}$ does. Thus, replacing the elements of $\tilde{\xi}_{\sigma}^{-}$with (7) gives us coordinates that are invariant under $\sigma$. We begin this procedure with the reduction of $T_{x}\left(L_{x} / 2\right)$, and repeat for $T_{z}\left(L_{z} / 2\right)$ and $R_{x y}$ to obtain the 8-to- 1 transformation to the symmetry-reduced coordinates $\xi$.

At the first stage of our study, similar to Refs. [8, 10], we generated turbulent data sets from random initial conditions with a total run time of 5864 and initiated Newton-Krylov-hookstep searches for POs from near-recurrences of the turbulent flow as measured by $R\left(t, t^{\prime}\right)=\left\|\mathbf{u}\left(t+t^{\prime}\right)-\mathbf{u}(t)\right\| /\|\mathbf{u}(t)\|$, where $\|\mathbf{u}\|^{2}=$ $\frac{1}{L_{x} L_{y} L_{z}} \int \mathbf{u} \cdot \mathbf{u} d \mathbf{x}$. With the choices of recurrence threshold $R_{t h}=0.3$ for triggering PO searches and recurrence time $t^{\prime} \in[0,20]$, this process resulted in 18 distinct POs with relative errors $\left\|\mathbf{u}_{p}\left(t+T_{p}\right)-\mathbf{u}_{p}(t)\right\| /\left\|\mathbf{u}_{p}(t)\right\|$ less than $10^{-9}$. We found two of these POs to have very similar physical properties and thus discarded one of them to retain 17. This omission had no significant effect on our results [29]. Hereafter, we refer to these orbits as $\overline{\mathrm{po}}_{i}$ with indices $i=1,2, \ldots, 17$ ordered in increasing periods, where the shortest period $T_{1}=2.8$ and the longest one $T_{17}=17.3$ [29].

The first question that we address is how frequently individual POs are visited, i.e. shadowed, by the turbulent flow. Our analysis begins with producing projection bases for individual POs. To this end, we take snapshots along one period of each orbit with the sampling time $t_{s}=0.1$ and generate the associated principal components [32] in the symmetry-reduced state space using the $L_{2}$ inner product $\left\langle\xi^{(k)}, \xi^{(l)}\right\rangle=\sum_{i} \xi_{i}^{(k)} \xi_{i}^{(l)}$. Next, we simulate turbulent flow and project it onto each of these bases centered at the empirical mean of the respective PO. As an illustration, Fig. 1(a) shows $\overline{\mathrm{po}}_{13}$ along with a shadowing turbulent trajectory spanning a time window equal to the period $T_{13}=14.8$ of $\overline{\mathrm{po}}_{13}$ as projections onto the leading three principal components of $\overline{\mathrm{po}}_{13}$. The main idea of state space persistence analysis [17] is quantifying the shape similarity of projections of the POs and those of turbulent trajectories such as the ones shown in Fig. 1(a). For this purpose, we utilize persistent homology, which we briefly describe next and refer to Refs. [33-35] for in-depth introductions.

Persistent homology is a computational topology 

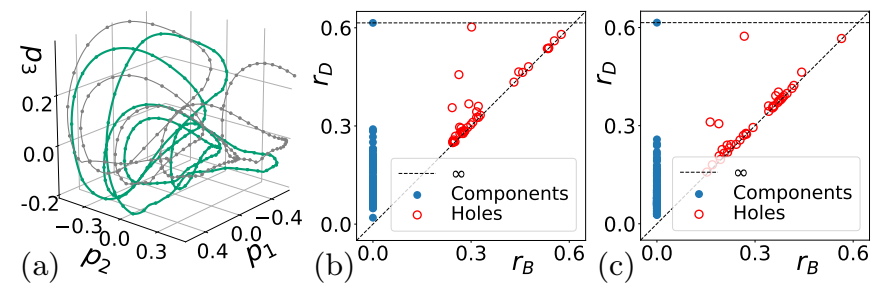

FIG. 1. (Color online) (a) $\overline{\mathrm{po}}_{13}$ (green/thick) and a shadowing trajectory (gray/thin) visualized as projections onto the leading three principal components of $\overline{\mathrm{po}}_{13}$. (b,c) The persistence diagrams associated with $\overline{\mathrm{po}}_{13}$ (b) and shadowing trajectory segment (c) shown in (a). The data points used for generating the persistence diagrams $(b, c)$ are marked with dots along the projection curves in (a).

method for extracting shape information from a data set by generating its representations at different resolutions and tracking the topological changes in the process. In our applications, the data sets of interest are the state space projections such as those visualized in Fig. 1(a) and the final products of the persistence computation are the persistence diagrams, examples of which are shown in Fig. 1(b,c). Each marker in a persistence diagram corresponds to the birth and death of a topological feature represented by the pair $\left(r_{B}, r_{D}\right)$ of birth and death resolutions. For every data set $\Xi$, persistent homology gives us two diagrams $\mathrm{PD}_{0}$ and $\mathrm{PD}_{1}$ corresponding to the components and holes, respectively [36]. What is gained in this process is a way of quantifying the shape similarity since one can define a metric in the space of persistence diagrams. Assuming each diagram also contains the trivial elements at the diagonal $r_{B}=r_{D}$ with infinite multiplicity, we can define the bottleneck distance between $\mathrm{PD}^{(k)}$ and $\mathrm{PD}^{(l)}$ as

$$
W_{\infty}\left(\mathrm{PD}^{(k)}, \mathrm{PD}^{(l)}\right)=\inf _{\phi} \sup _{\mu \in \mathrm{PD}^{(k)}}\|\mu-\phi(\mu)\|_{\infty},
$$

where $\phi: \mathrm{PD}^{(k)} \rightarrow \mathrm{PD}^{(l)}$ is a bijection from $\mathrm{PD}^{(k)}$ to $\mathrm{PD}^{(l)}$. The bottleneck distance (8) can be interpreted as the largest (measured in the $L_{\infty}$ norm) of the shortest one-to-one pairings of the elements of $\mathrm{PD}^{(k)}$ and $\mathrm{PD}^{(l)}$. An important property of persistent homology that motivates our application is stability [37]: Small perturbations to the underlying data result in small variations, measured by the bottleneck distance (8), of the associated persistence diagrams.

We are now in position to define the shadowing distance. Let $\Xi^{\overline{\mathrm{po}}_{i}}=\left\{\hat{\xi}^{\overline{\mathrm{po}}_{i}}(0), \hat{\xi}^{\overline{\mathrm{po}}_{i}}\left(t_{s}\right), \ldots, \hat{\xi}^{\overline{\mathrm{po}}_{i}}\left(\left(N_{i}-1\right) t_{s}\right)\right\}$, and $\Xi^{(i)}(t)=\left\{\hat{\xi}(t), \hat{\xi}\left(t+t_{s}\right), \ldots, \hat{\xi}\left(t+\left(N_{i}-1\right) t_{s}\right)\right\}$ be states sampled at rate $t_{s}^{-1}$ along one period of $\overline{\mathrm{po}}_{i}$ and a chaotic trajectory beginning at time $t$, respectively, and ${ }^{\wedge}$ indicate the projection onto the bases of $\overline{\mathrm{po}}_{i}$. We define the shadowing distance $S^{(i)}(t)$ of turbulence from $\overline{\mathrm{po}}_{i}$ at time $t$ as

$$
S^{(i)}(t)=\sum_{k=0}^{1} w_{k} W_{\infty}\left(\mathrm{PD}_{k}^{(i)}(t), \mathrm{PD}_{k}^{\left(\overline{\mathrm{po}}_{i}\right)}\right)
$$

where $\mathrm{PD}^{\left(\overline{\mathrm{po}}_{i}\right)}$ and $\mathrm{PD}^{(i)}(t)$ are the persistence diagrams obtained from $\Xi^{\overline{\mathrm{Po}}_{i}}$ and $\Xi^{(i)}(t)$, respectively, and $w_{0,1}$ are the weights of respective contributions from the components and holes. In what follows, these weights are set to $w_{0,1}=\left[W_{\infty}\left(\mathrm{D}, \mathrm{PD}_{0}^{\left(\overline{\mathrm{po}}_{i}\right)}\right)+W_{\infty}\left(\mathrm{D}, \mathrm{PD}_{1}^{\left(\overline{\mathrm{p}}_{i}\right)}\right)\right]^{-1}$, where $\mathrm{D}$ denotes the empty persistence diagram with diagonal elements only. This choice of the weights sets the shadowing distance of a PO to an empty data set to 1; thus renders the shadowing distances from different POs comparable. As an illustration, Fig. 2(a) shows the shadowing distances of a turbulent trajectory from 8 out of 17 POs.

We expect the local minima of $S^{i}(t)$ to correspond to the episodes of turbulent flow shadowing $\overline{\mathrm{po}}_{i}$. Following this assumption, we define the shadowing decomposition of a turbulent flow in a time interval $t \in\left[t_{0}, t_{f}\right]$ over $\left\{\overline{\mathrm{po}}_{1}, \overline{\mathrm{po}}_{2}, \ldots \overline{\mathrm{po}}_{N_{\overline{\mathrm{po}}}}\right\}$ for a threshold distance $S_{\mathrm{th}}$ by the following algorithm. Starting at time $t=t_{0}$, we find $i_{\min }=\arg \min _{i} S_{i}(t)$. If $S_{i_{\min }}(t)$ is less than $S_{t h}$, then we save the pair $\left(t, i_{\min }\right)$ and increase $t$ by $T_{i_{\min }}$; otherwise, we increase $t$ by $t_{s}$ and repeat the procedure until the final time $t_{f}$ is reached. The result is the set of pairs $\left(t, i_{\min }\right)$ which we interpret as "turbulence at time interval $\left[t, t+T_{i_{\min }}\right]$ can be approximated by $\overline{\mathrm{po}}_{i_{\min }}$." In Fig. 2(b), we visualized the shadowing decomposition $\left(S_{\mathrm{th}}=0.5\right)$ of turbulence corresponding to the same episode as Fig. 2(a) as a bar plot where the length of each bar is equal to the period of the respective PO. Supplementary video [29] shows another visualization of this decomposition for $t \in[0,100]$ where velocity and vorticity isosurfaces of turbulence are shown next to those of the POs that are being shadowed along with their state space projections. As can be seen in the supplementary video (also demonstrated in SM [29]), our decomposition is able to generate shadowing signals even when turbulence follows a PO for less than a full period.

From its shadowing decomposition, we can infer a model of the turbulent flow as a Markov chain [38] with the transition matrix $P$, whose elements $P_{i j}$ correspond to the probability of shadowing $\overline{\mathrm{po}}_{j}$ after $\overline{\mathrm{po}}_{i}$. We estimated these probabilities from 18 different runs, separate from those used to find the POs, with a total run time of $t_{\text {tot }}=25039$ excluding the initial transients and the laminarization events [29]. For the threshold choice $S_{\mathrm{th}}=0.5$, we found the shadowing events to cover $75 \%$ of the total time. As a robustness test, we repeated our computations for $S_{\text {th }} \in[0.4,0.6]$. While the fraction of turbulent time that is covered by the POs differs for different $S_{\mathrm{th}}$, it remains always above $50 \%$ ( $54 \%$ for $S_{\mathrm{th}}=0.4$ and $88 \%$ for $\left.S_{\mathrm{th}}=0.6\right)$ and the transition probabilities of the Markov process vary only slightly [29]. Therefore, our results in 


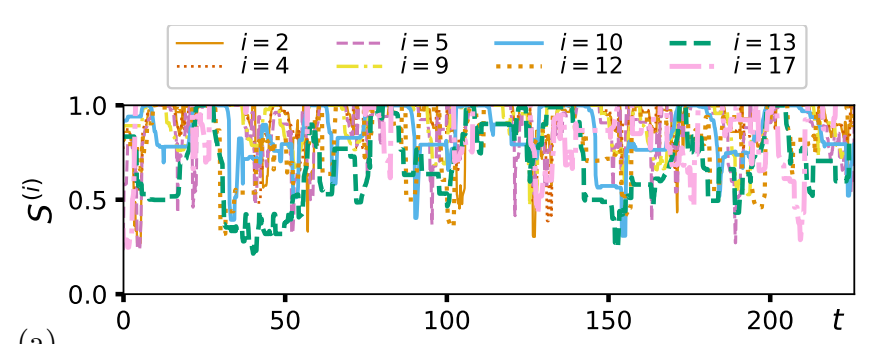

(a)

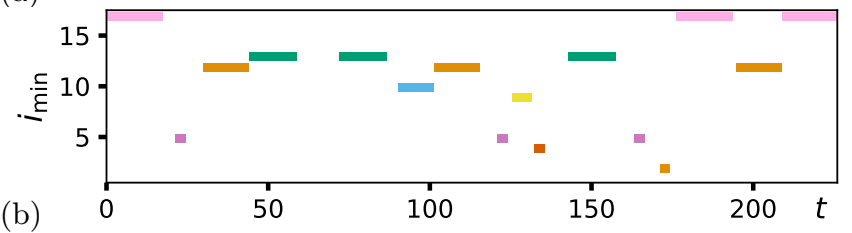

(c)

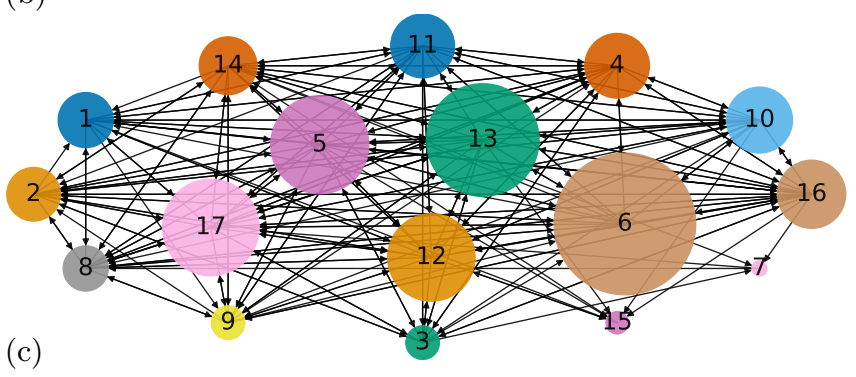

FIG. 2. (Color online) (a) Shadowing distance of a turbulent trajectory from 8 POs. (b) Shadowing decomposition of the turbulent trajectory. (c) State transition graph where the nodes correspond to POs and arrows indicate the possible transitions between them. The self-loops are omitted for clarity and the node sizes are proportional to the probability of observing the respective PO as inferred from the invariant distribution of the corresponding Markov process.

the following are not sensitive to this threshold.

The invariant distribution $\pi$ of the Markov chain is the left eigenvector of $P$ with unit eigenvalue, satisfying the normalization condition $\sum_{i} \pi_{i}=1$. Fig. 2(c) shows a network visualization of the Markov chain that models the $3 D$ Kolmogorov flow that we studied here. Each node of Fig. 2(c) corresponds to a PO with the size of the node $i$ proportional to $\pi_{i}$ and the directed edges indicate possible transitions from one PO to the next. The nodes have also self-loops (not shown in Fig. 2(c) for clarity) that correspond to close recurrence events in which turbulence shadows a PO for more than one period. From $\pi$, we can predict long-time averages of the turbulent flow's observables in terms of their values computed over POs. Let $\Omega$ be an observable and $\langle\Omega\rangle_{i}$ be its average over $\overline{\mathrm{po}}_{i}$ and its symmetry copies. The long-time average $\langle\Omega\rangle_{\infty}$ can be approximated as

$$
\langle\Omega\rangle_{\pi}=\frac{\sum_{i=1}^{N_{\overline{\mathrm{DO}}}} \pi_{i} T_{i}\langle\Omega\rangle_{i}}{\sum_{i=1}^{N_{\overline{\mathrm{DO}}}} \pi_{i} T_{i}},
$$

where we interpret the coefficients $\pi_{i} T_{i}$ as the mean time that chaotic flow spends in the neighborhood of $\overline{\mathrm{po}}_{i}$. The observables that we consider are kinetic energy $E=\|\mathbf{u}\|^{2} / 2$, power input $I=\frac{1}{L_{x} L_{y} L_{z}} \int \mathbf{u} \cdot \mathbf{f} d \mathbf{x}$, dissipation $D=\nu\|\nabla \times \mathbf{u}\|^{2}$, and the velocity profile $U(y)=\frac{1}{L_{x} L_{z}} \iint u(x, y, z) d x d z$. Fig. $3(\mathrm{a}, \mathrm{b})$ show the POs and the data sampled from turbulence on $I D$ and $E \dot{E}$ planes $(\dot{E}=I-D)$ respectively. The long-time averages $\langle D\rangle_{\infty}=\langle I\rangle_{\infty}=1.885,\langle E\rangle_{\infty}=10.54$, and $\langle\dot{E}\rangle_{\infty}=0$ along with the PO estimates $(10)\langle D\rangle_{\pi}=$ $\langle I\rangle_{\pi}=1.874,\langle E\rangle_{\pi}=10.85$, and $\langle\dot{E}\rangle_{\pi}=0$ are also marked in Fig. 3(a,b). In Fig. 3(c), we plot the mean PO velocity profiles along with the long-time average $\langle U(y)\rangle_{\infty}$ and its PO estimate $\langle U(y)\rangle_{\pi}$ (10). These longtime $\left(t_{\text {total }}=35492\right)$ averages are computed over runs that are separate from those used to infer the transition probabilities.
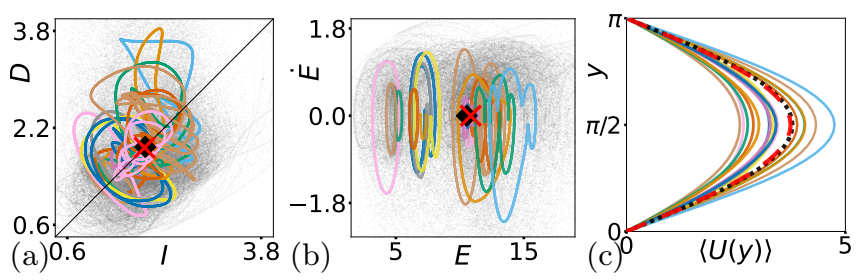

FIG. 3. (Color online) Turbulent trajectories (dots, gray) and POs (loops, colors) visualized (a) on the $I D$ and (b) the $E \dot{E}$ planes along with the long-time averages $\langle D\rangle_{\infty}=\langle I\rangle_{\infty}=$ $1.885,\langle E\rangle_{\infty}=10.54$, and $\langle\dot{E}\rangle_{\infty}=0$ (diamonds, black) and the PO estimates $\langle D\rangle_{\pi}=\langle I\rangle_{\pi}=1.874,\langle E\rangle_{\pi}=10.85$, and $\langle\dot{E}\rangle_{\pi}=0$ (crosses, red). (c) Velocity profiles averaged over POs (solid, colors) along with the long-time average $\langle U(y)\rangle_{\infty}$ (dotted, black) and its PO estimate $\langle U(y)\rangle_{\pi}$ (dashed, red). Only the half domain $y \in[0, \pi]$ is shown in (c) since the other half corresponds to its mirror image.

As noted above and shown in Fig. 3, the PO estimates (10) of observables agree (to 2 digits for $E, I$, and $D$ ) with the long-time averages, providing an a posteriori verification of our reduced-order model. It is worth emphasizing that the observable averages over individual POs can be quite different (Fig. 3, see also [29]) from the long-time averages. Thus, it is crucial for the weights in the sum (10) to be correct for numerical agreement. Interestingly, we found the orbits with long periods to be necessary to capture the long-time averages since our shortest 9 POs with periods less than 10 have mean dissipation rates less than the long-time average $\langle D\rangle_{\infty}=1.885$ [29]. This observation is at odds with our intuition based on the cycle expansions of strange sets [39] where the long POs appear only in correction terms.

One feature of the $3 D$ Kolmogorov flow that we do not capture in our model is the laminarization events since we exclude them from our training data. Therefore, the Markov chain and its invariant distribution should be understood as the model of the nonattracting chaotic set [20] underlying transient turbulence and the natural measure over it, respectively. We note that the consistency of the long-time averages with those computed using (10) is evidence of ergodicity for this chaotic set.

In this study, we combined ideas from the dynamical 
systems theory with topological data analysis to produce a low-dimensional turbulence model, wherein the dynamics is viewed as a Markov chain of shadowing events. We confirmed the accuracy of this description by reproducing the long-time averages of the flow's observables from the invariant distribution of the Markov process. We would like to note that coarse-grained models such as ours can be utilized in control methods that drive the system towards desired state space regions. In conclusion, we believe that modeling turbulence using POs not only deepens our understanding of it but also opens new avenues for applications.

We thank the referees for improving this paper with their comments. We acknowledge stimulating discussions with H. Edelsbrunner. This work was supported by a grant from the Simons Foundation (662960, BH). The numerical calculations were performed at TUBITAK ULAKBIM High Performance and Grid Computing Center (TRUBA resources) and IST Austria High Performance Computing cluster.

[1] M. Nagata, J. Fluid Mech. 217, 519 (1990).

[2] F. Waleffe, Phys. Rev. Lett. 81, 4140 (1998).

[3] G. Kawahara and S. Kida, J. Fluid Mech. 449, 291 (2001).

[4] H. Faisst and B. Eckhardt, Phys. Rev. Lett. 91, 224502 (2003), arXiv:nlin/0304029 [nlin.CD].

[5] H. Wedin and R. R. Kerswell, J. Fluid Mech. 508, 333 (2004).

[6] B. Hof, C. W. H. van Doorne, J. Westerweel, F. T. M. Nieuwstadt, H. Faisst, B. Eckhardt, H. Wedin, R. R. Kerswell, and F. Waleffe, Science 305, 1594 (2004).

[7] J. F. Gibson, J. Halcrow, and P. Cvitanović, J. Fluid Mech. 611, 107 (2008), arXiv:0705.3957 [physics.flu-dyn].

[8] P. Cvitanović and J. F. Gibson, Phys. Scr. T142, 014007 (2010).

[9] L. van Veen and G. Kawahara, Phys. Rev. Lett. 107, 114501 (2011), arXiv:1103.1331 [physics.flu-dyn].

[10] N. B. Budanur, K. Y. Short, M. Farazmand, A. P. Willis, and P. Cvitanović, J. Fluid Mech. 833, 274 (2017), arXiv:1705.03720 [physics.flu-dyn].

[11] N. B. Budanur, A. S. Dogra, and B. Hof, Phys. Rev. Fluids 4, 102401 (2019), arXiv:1810.02211 [physics.fludyn].

[12] E. Hopf, Commun. Pure Appl. Math. 1, 303 (1948).

[13] N. B. Budanur and B. Hof, Phys. Rev. Fluids 3, 054401 (2018), arXiv:1802.01918 [physics.flu-dyn].

[14] D. Auerbach, P. Cvitanović, J.-P. Eckmann, G. Gunaratne, and I. Procaccia, Phys. Rev. Lett. 58, 23 (1987).

[15] D. Viswanath, J. Fluid Mech. 580, 339 (2007), arXiv:physics/0604062 [physics.flu-dyn].

[16] J. V. Shebalin and S. L. Woodruff, Phys. Fluids 9, 164 (1997).
[17] G. Yalnız and N. B. Budanur, Chaos 30, 033109 (2020), arXiv:1910.04584 [nlin.CD].

[18] P. Gaspard, Chaos, Scattering and Statistical Mechanics (Cambridge Univ. Press, Cambridge, 1998).

[19] P. Cvitanović, R. Artuso, R. Mainieri, G. Tanner, and G. Vattay, Chaos: Classical and Quantum (Niels Bohr Inst., Copenhagen, 2017) webbook, stable version 15.9, ChaosBook.org.

[20] Y. Lai and T. Tél, Transient Chaos: Complex Dynamics on Finite Time Scales (Springer, New York, 2011).

[21] L. van Veen and S. Goto, Fluid Dyn. Res. 48, 061425 (2016), arXiv:1512.02570 [physics.flu-dyn].

[22] P. Manneville, Mech. Eng. Rev. 3, 15 (2016), arXiv:1604.00840 [physics.flu-dyn].

[23] G. Yalnız and N. B. Budanur, dnsbox code \& data, github.com/burakbudanur/dnsbox (2021), Periodic orbit data along with our DNS code will be available in this repository.

[24] S. A. Orszag, Phys. Fluids 12, II (1969).

[25] C. Canuto, M. Y. Hussaini, A. Quarteroni, and T. A. Zang, Spectral Methods: Evolution to Complex Geometries and Applications to Fluid Dynamics (Springer, New York, 2007).

[26] S. G. Chumakov, Phys. Fluids 19, 058104 (2007).

[27] A. P. Willis, SoftwareX 6, 124 (2017), arXiv:1705.03838 [physics.flu-dyn].

[28] N. Saul and C. Tralie, Scikit-tda: Topological data analysis for python (2019).

[29] See Supplemental Material at [URL will be inserted by publisher] for details on the (i) adequacy of our resolution, (ii) effective number of degrees of freedom, (iii) POs and their selection for modeling, (iv) demonstration of partial shadowing of a PO, (v) robustness against threshold choice, and (vi) convergence of statistics and models.

[30] N. B. Budanur, P. Cvitanović, R. L. Davidchack, and E. Siminos, Phys. Rev. Lett. 114, 084102 (2015), arXiv:1405.1096 [nlin.CD].

[31] N. B. Budanur and P. Cvitanović, J. Stat. Phys. 167, 636 (2017), arXiv:1509.08133 [nlin.CD].

[32] I. T. Jolliffe, Principal Component Analysis, Second Edition (Springer, 2002).

[33] H. Edelsbrunner and J. Harer, Persistent homology-a survey, in Surveys on Discrete and Computational Geometry: Twenty Years Later (Am. Math. Soc., Providence, 2008) pp. 257-282.

[34] C. Epstein, G. Carlsson, and H. Edelsbrunner, Inverse Probl. 27, 120201 (2011).

[35] N. Otter, M. A. Porter, U. Tillmann, P. Grindrod, and H. A. Harrington, EPJ Data Sci 6, 17 (2017), arXiv:1506.08903 [math.AT].

[36] In general, higher-dimensional voids are included in persistence diagrams, however, in our applications we consider one-dimensional holes only.

[37] D. Cohen-Steiner, H. Edelsbrunner, and J. Harer, Discrete Comput Geom. 37, 103 (2007).

[38] A. Papoulis and S. U. Pillai, Probability, Random Variables, and Stochastic Processes (McGraw-Hill, 2002).

[39] P. Cvitanović, Phys. Rev. Lett. 61, 2729 (1988). 


\section{Supplemental Material}

\section{NUMERICAL REPRESENTATION AND THE ADEQUACY OF RESOLUTION}

We represent the velocity fields as a Fourier expansion

$$
\mathbf{u}(x, y, z)=\sum_{k_{x}, k_{y}, k_{z}} \hat{\mathbf{u}}\left[k_{x}, k_{y}, k_{z}\right] e^{i\left(k_{x} x+k_{y} y+k_{z} z\right)},
$$

where the sum is carried through all resolved wave numbers. In our $[2 \pi \times 2 \pi \times \pi]$ domain with $[64 \times 64 \times 32]$ grid points, after the $2 / 3$ dealiasing, the nonzero wave numbers in $x, y$, and $z$ directions are

$$
\begin{aligned}
& k_{x}=\{-20,-19, \ldots, 19,20\}, \\
& k_{y}=\{-20,-19, \ldots, 19,20\}, \\
& k_{z}=\{-20,-18, \ldots, 18,20\} .
\end{aligned}
$$

In the literature, the energy spectrum of turbulent flows is usually presented in the time- and shell-averaged form as shown in Fig. S1(a), where $E_{k}$ is the total energy contained in Fourier coefficients with amplitudes falling in the interval $[k, k+1)$. Note that the wave numbers shown in Fig. S1(a) go beyond the largest resolved wave number $k_{\max }=20$ in each direction since the amplitude of the wave vector $\left[k_{x}=20, k_{y}=20, k_{z}=20\right]$ is $|\mathbf{k}|_{\max } \approx 34.64$. In order to demonstrate the adequacy of our resolution, we therefore show the time-averaged spectra individually for each spatial direction in Fig. S1(b), where one can see at least 6 orders of magnitude drop-off from the longest resolved wave length to the shortest one. Even though the time-averaged spectra of Fig. S1 show 6 orders of magnitude or more drop-off, we observed that this spectral gap can be as low as 4 orders instantaneously for states with high dissipation. We show the instantaneous energy spectra for one such state in Fig. S1(c).
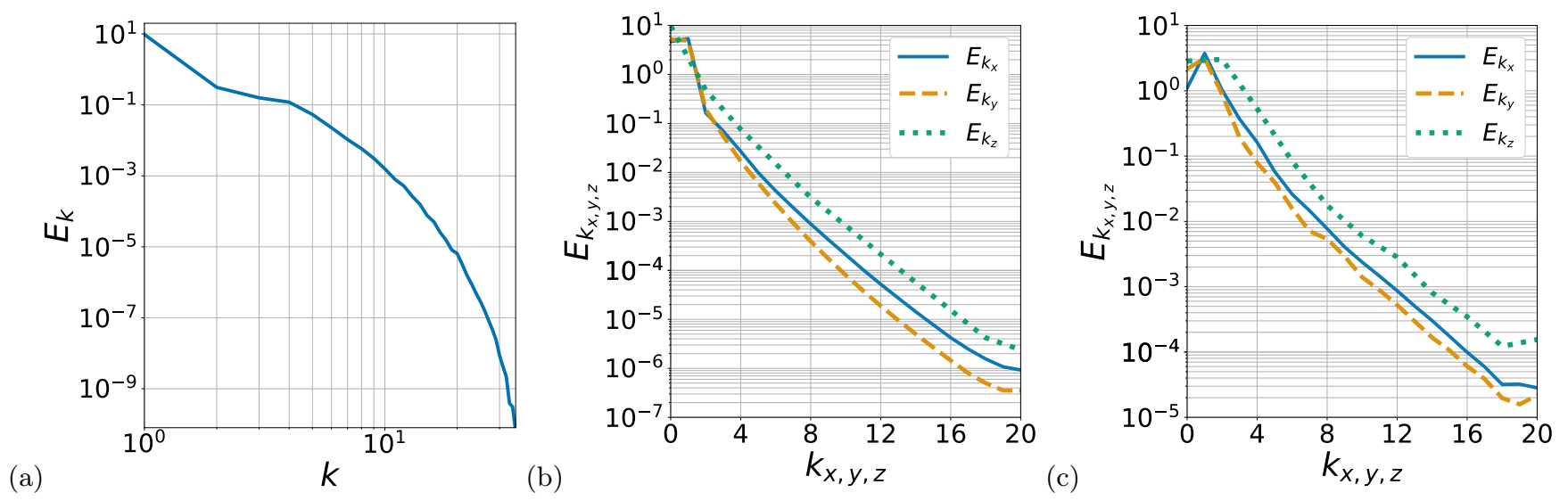

FIG. S1. (a) Time- and shell-averaged energy spectrum. (b) Time-averaged energy spectra in each spatial direction. The time averages are obtained by averaging the spectrum of 3012 distinct turbulent state sampled every $\Delta t=40$ from 21 runs. (c) Example of a "worst-case" energy spectra where the spectral drop-off in $x$ direction is 4 orders of magnitude.

As a further test of the adequacy of our numerical representation, we progressively increased the spatial and temporal resolution up to a factor of $3 / 2$ and confirmed that our periodic orbits continue to exist at these higher resolutions by Newton-converging them at every step. In our preliminary work where we used a lower spatial resolution $\left[N_{x}=32, N_{y}=32, N_{z}=16\right]$, some of the periodic orbits that we found did not pass this test at various intermediate steps leading us to our final spatial resolution $[64,64,32]$.

Our spatial resolution is slightly lower than that of Ref. [21], where the authors reported their results using the Reynolds number $R e=L_{y} \sqrt{L_{y} \gamma} / 2 \pi \nu$ as the control parameter. Following this definition, our choice of parameters yields $R e \approx 50$, which is much lower than $R e=170$ at which Ref. [21] reports long-lived turbulent transients. At first glance, our observation of long-lived turbulent transients at $R e \approx 50$ might seem to contradict with the results of Ref. [21]. However, the possibility of observing turbulence in this system at $R e$ as low as $R e=40$ was already reported in the discussion of Ref. [21], where the authors speculated the existence of edge states other than the one they reported. We confirmed their observation by matching our domain exactly to theirs by extending the spatial 
extent in the $z$ direction to $L_{z}=2 \pi$ and lifting the symmetry constraints that we impose on the dynamics. Our numerical experiments indicate that it is possible to observe long-lived turbulent transients with life times of $O(1000)$ in such domains.

\section{EFFECTIVE NUMBER OF DEGREES OF FREEDOM}

The wave numbers that are kept in our numerical integrator at $L_{x} \times L_{y} \times L_{z}=2 \pi \times 2 \pi \times \pi$, excluding dealiased modes, are

$$
\begin{aligned}
& k_{x}=\{-20,-19, \ldots, 19,20\}, \\
& k_{y}=\{-20,-19, \ldots, 19,20\}, \\
& k_{z}=\{0,2, \ldots, 18,20\},
\end{aligned}
$$

where we omit the $k_{z}<0$ part of the spectrum since they can be recovered from the condition $\hat{\mathbf{u}}^{*}[-\mathbf{k}]=\hat{\mathbf{u}}[\mathbf{k}]$ the expansion coefficients obey as the velocity field in the physical space is real valued. This gives $41 \times 41 \times 11 \times 2 \times 3=$ 110946 numerical degrees of freedom, where the factors of 2 and 3 correspond to real and imaginary parts of the Fourier coefficients and the dimensions of physical space, respectively. Although this discretization implies a 110946dimensional dynamical system, in practice, the number of degrees of freedom in our system is effectively reduced by the divergence-free condition and the imposed symmetries. Since the $\mathbf{k}=0$ mode is time-invariant (Galilean invariance) we set it to 0 , which eliminates 6 degrees of freedom from the Fourier series. The divergence-free condition $i \mathbf{k} \cdot \hat{\mathbf{u}}=0$ reduces the independent number of degrees of freedom by a factor of $2 / 3$, since the knowledge of two velocity field components uniquely determines the third one. In addition, restricting the dynamics into a subspace that is invariant under the symmetries $S_{x}$ and $R_{z}$ further drop the degrees of freedom by a factor of 4 , resulting in the final number of independent degrees of freedom $[(110946-6) \times 2 / 3] / 4=18490$.

The number of independent numerical degrees of freedom is still much higher than the manifold in which the turbulent dynamics takes place. Although, we do not have a rigorous proof of existence of such an inertial manifold, we think that it is reasonable to assume its existence due to the dissipation in the system. While estimating the dimension of this manifold is beyond the scope of the present work, we here provide an evaluation of various lowerdimensional embeddings based on principal component analysis. To this end, we first compute 24096 principal components corresponding to 3012 uncorrelated turbulent states and their 8 discrete symmetry copies. We then construct embeddings of different dimensions using the leading $d$ principal components and compute the error

$$
\epsilon_{\mathrm{PCA}}=\|\mathbf{u}-\mathbf{P u}\| /\|\mathbf{u}\|
$$

where $\mathbf{u}$ is sampled from a test set distinct from those that were used to construct the principal components and $\mathbf{P}$ denotes the projection onto the principal components. Table SI shows the minimum, maximum, and mean errors on this test set for different embedding dimensions.

TABLE SI. Minimum, maximum, and mean error (S4) for different embedding dimensions computed for a test turbulent trajectory with a lifetime $t=1924.0$, sampled at $t_{s}=0.1$.

\begin{tabular}{cccc}
\hline \hline$d$ & $\min \epsilon_{\mathrm{PCA}}$ & $\max \epsilon_{\mathrm{PCA}}$ & $\left\langle\epsilon_{\mathrm{PCA}}\right\rangle$ \\
\hline 64 & $1.224 \times 10^{-2}$ & 0.3884 & 0.07880 \\
128 & $6.006 \times 10^{-3}$ & 0.2775 & 0.05151 \\
256 & $2.866 \times 10^{-3}$ & 0.1774 & 0.03101 \\
512 & $1.150 \times 10^{-3}$ & 0.1188 & 0.01696 \\
1024 & $4.468 \times 10^{-4}$ & 0.07208 & 0.008266 \\
2048 & $1.593 \times 10^{-4}$ & 0.03930 & 0.003540 \\
4096 & $4.393 \times 10^{-5}$ & 0.02005 & 0.001268 \\
\hline \hline
\end{tabular}

\section{SELECTION OF THE PERIODIC ORBITS}

In Table SII we list the period, mean kinetic energy and dissipation, and the contribution to the invariant distribution of the 18 periodic orbits that we found from near recurrences of the turbulent flow. We began our modeling trials 
using the subset of periodic orbits with periods shorter than 10, however, all of these attempts resulted in estimates of dissipation lower than its long-time average. The reason behind this is readily seen in Table SII where all of the periodic orbits with $T<10$ have mean rate of dissipation less than the long-time average $\langle D\rangle_{\infty}=1.885$. Consequently, we decided to use all numerically-found periodic orbits. However, as we shall explain in the following, we found that excluding $\overline{\mathrm{po}}_{18}$ from the model did not result in a significant change in our final results.

TABLE SII. List of periodic orbits. Shown are the period $T$, average kinetic energy $\langle E\rangle$, and average dissipation $\langle D\rangle$, and contribution to the invariant distribution $\pi$.

\begin{tabular}{ccccc}
\hline \hline $\mathrm{i}$ & $T$ & $\langle E\rangle$ & $\langle D\rangle$ & $\pi$ \\
\hline 1 & 2.8076 & 10.6017 & 1.6967 & $3.3392 \times 10^{-2}$ \\
2 & 2.9285 & 7.1407 & 1.5095 & 1.4172 \\
3 & 3.0481 & 5.2235 & 1.4752 & $1.2416 \times 10^{-2}$ \\
4 & 3.2001 & 6.3834 & 1.6435 & $4.6056 \times 10^{-2}$ \\
5 & 3.2027 & 10.4267 & 1.3260 & $1.0589 \times 10^{-1}$ \\
6 & 3.3281 & 4.6274 & 1.4045 & $2.2066 \times 10^{-1}$ \\
7 & 4.8178 & 4.2158 & 1.6826 & $2.2472 \times 10^{-2}$ \\
8 & 5.7962 & 7.1860 & 1.7262 & $1.2433 \times 10^{-2}$ \\
9 & 5.9469 & 7.5110 & 2.3542 & $4.7897 \times 10^{-2}$ \\
10 & 11.1259 & 14.2693 & 1.6708 & 2.0303 \\
11 & 11.9670 & 7.1459 & 2.0253 & $8.4907 \times 10^{-2}$ \\
12 & 14.0560 & 12.2741 & 1.9465 & $1.3957 \times 10^{-2}$ \\
13 & 14.8255 & 12.7118 & 1.6996 & $3.6768 \times 10^{-1}$ \\
14 & 15.0668 & 12.0885 & 2.1675 & $5.4522 \times 10^{-3}$ \\
15 & 15.2772 & 11.1163 & 1.7239 & $1.1218 \times 10^{-2}$ \\
16 & 16.5225 & 11.9875 & 1.7930 & 0 \\
17 & 17.3382 & 10.6745 & & $1.0258 \times 10^{-1}$ \\
\hline \hline
\end{tabular}

Since our recurrence-based periodic orbit search is an experimental process, it is reasonable to expect some periodic orbits to be located nearby in the state space with similar physical properties. We search for such cases by defining a periodic orbit shadowing distance as follows. Let $\Xi^{\left(i, \overline{\mathrm{po}}_{j}\right)}=\left\{\hat{\xi}^{\overline{\mathrm{po}}_{j}}(0), \hat{\xi}^{\overline{\mathrm{po}}_{j}}\left(t_{s}\right), \ldots, \hat{\xi}^{\overline{\mathrm{po}}_{j}}\left(\left(N_{j}-1\right) t_{s}\right)\right\}$ be the projection of the states sampled on the $\overline{\mathrm{po}}_{j}$ onto the bases of $\overline{\mathrm{po}}_{i}$. We define the shadowing distance of $\overline{\mathrm{po}}_{j}$ from $\overline{\mathrm{po}}_{i}$ as

$$
S^{(i j)}=w_{0} W_{\infty}\left(\mathrm{PD}_{0}^{\left(i, \overline{\mathrm{po}}_{j}\right)}(t), \mathrm{PD}_{0}^{\left(\overline{\mathrm{po}}_{i}\right)}\right)+w_{1} W_{\infty}\left(\mathrm{PD}_{1}^{\left(i, \overline{\mathrm{po}}_{j}\right)}(t), \mathrm{PD}_{1}^{\left(\overline{\mathrm{po}}_{i}\right)}\right)
$$

where $\mathrm{PD}^{\left(i, \overline{\mathrm{po}}_{j}\right)}$ denotes the persistence diagrams associated with $\Xi^{\left(i, \overline{\mathrm{po}}_{j}\right)}$ and the weights $w_{0,1}$ are defined in the same way as in the shadowing distance of turbulence from $\overline{\mathrm{po}}_{i}$. We visualized $S^{(i j)}$ as a heat map in Fig. S2(a). By definition (S5), $S^{(i j)}$ is not symmetric under $i \rightleftarrows j$, which is also visible in Fig. S2(a). Nevertheless, if two periodic orbits $\overline{\mathrm{po}}_{i}$ and $\overline{\mathrm{po}}_{j}$ are located at nearby state space regions and possess similar shapes, we expect both $S^{(i j)}$ and $S^{(j i)}$ to be small. We found this to be the case for the pairs $(11,14)$ and $(17,18)$. While $\overline{\mathrm{po}}_{11}$ and $\overline{\mathrm{po}}_{14}$ have considerably different $\langle E\rangle$ and $\langle D\rangle$, those of $\overline{\mathrm{po}}_{17}$ and $\overline{\mathrm{po}}_{18}$ agree in two digits. In order to illustrate their similarity, we visualized $\overline{\mathrm{po}}_{17}$ and $\overline{\mathrm{po}}_{18}$ as projections onto the principal components associated with $\overline{\mathrm{po}}_{17}$ in Fig. S2(b). As a final test, we show the shadowing distances of a turbulent trajectory segment from $\overline{\mathrm{po}}_{17}$ and $\overline{\mathrm{po}}_{18}$ in Fig. S2(c), where one can see that the minima of $S^{17}(t)$ and $S^{18}(t)$ always appear near one another. Based on these observations, we decided to retain only one of these periodic orbits in our model. We confirmed that this choice had no effect in the first two digits of the observable averages computed from the invariant distribution of our Markov chain. We show the invariant distributions of the Markov chains with and without $\overline{\mathrm{po}}_{18}$ where one can see that the statistical weight of $\overline{\mathrm{po}}_{18}$ is transferred predominantly to $\overline{\mathrm{po}}_{17}$ when it is omitted. 


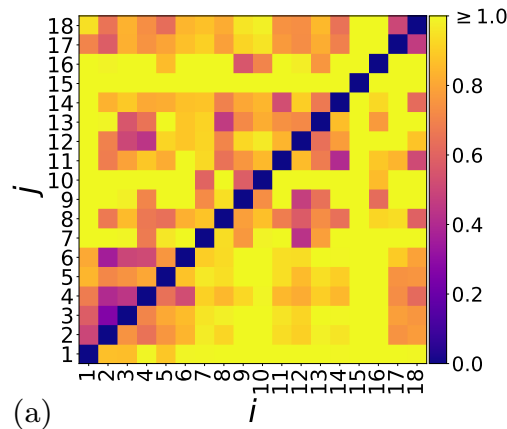

(a)

$i$

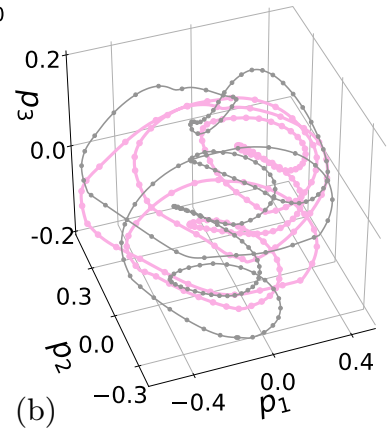

(b)

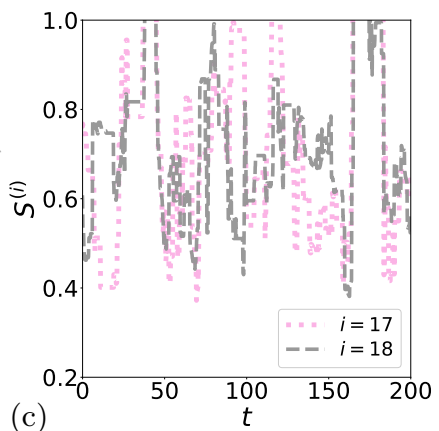

(c)

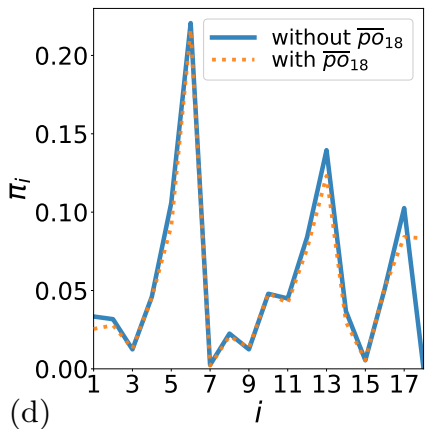

FIG. S2. (a) Heatmap showing the shadowing distances (S5) between periodic orbits. (b) Projections of $\overline{\mathrm{po}}_{17}$ (pink/thick) and $\overline{\mathrm{po}}_{18}$ (gray/thin) onto the first three principal components associated with $\overline{\mathrm{po}}_{17}$. (c) Shadowing distances $S^{(17,18)}(t)$ of a turbulent trajectory segment from $\overline{\mathrm{po}}_{17}$ and $\overline{\mathrm{po}}_{18}$. (d) Invariant distribution $(\pi)$ computed with (blue/thick) and without (yellow/dotted) $\overline{\mathrm{po}}_{18}$. The data points are connected with line segments for guiding the eye.

\section{SPATIAL STRUCTURES OF THE PERIODIC ORBITS}

Distributions of velocity gradients are often of interest in turbulent flows where heavy tails imply increased energy dissipation. Fig. S3 shows the distribution of gradients $\partial u_{i} / \partial x_{j}, i \neq j$ where the solid (dashed) lines correspond to POs with average dissipation greater (less) than the long-time average of turbulence. As shown, the difference of distributions is most pronounced in $\partial u / \partial y$ (Fig. S3(a)) and $\partial v / \partial x$ (Fig. S3(e)) terms where such solid curves appear to peak at higher values.
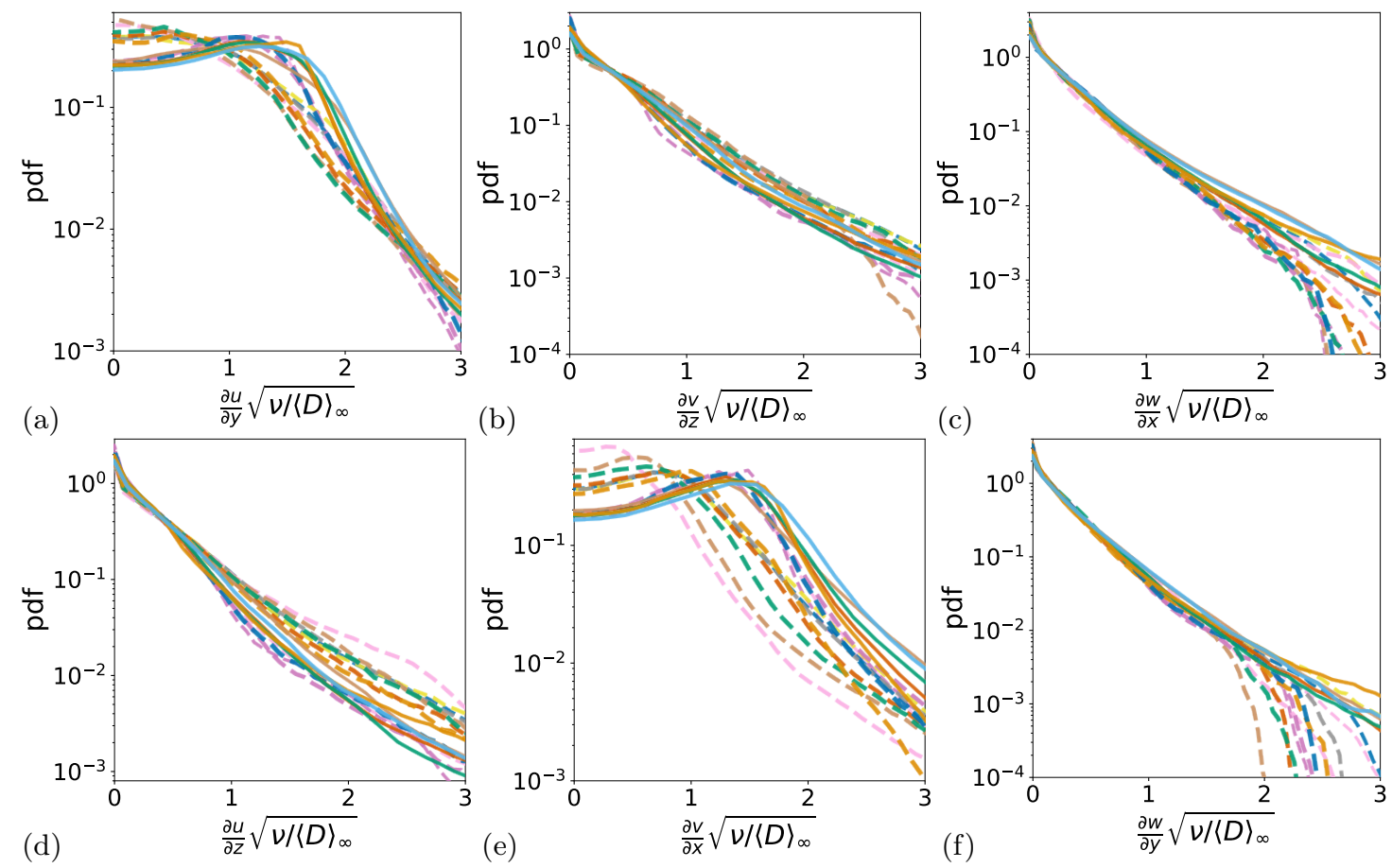

(b)

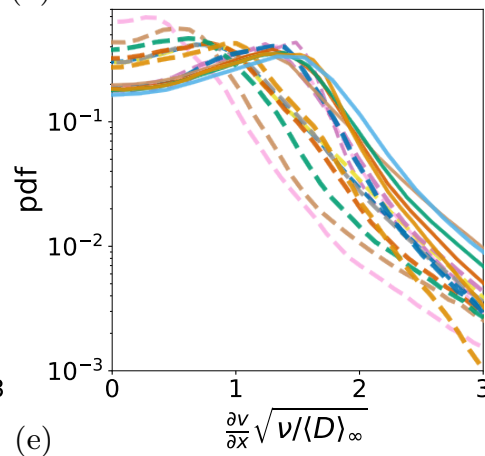

(c)

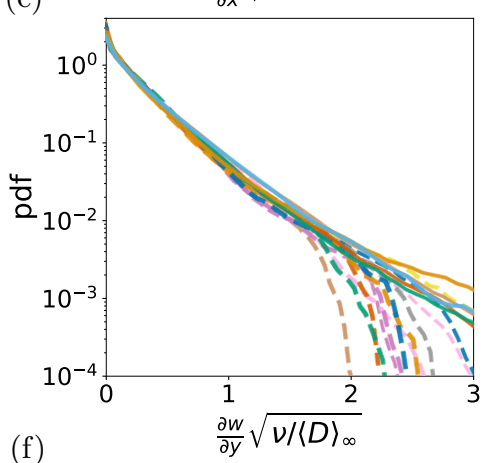

FIG. S3. Distributions of the gradients $\partial u_{i} / \partial x_{j}, i \neq j$ on the periodic orbits. Only the positive half is shown since the distributions are symmetric around 0. Solid (dashed) lines correspond to the periodic orbits with average dissipation greater (less) than $\langle D\rangle_{\infty}$. 


\section{PARTIAL SHADOWING OF PERIODIC ORBITS}

An important feature of our shadowing distance based on the shape similarity of turbulent trajectory segments and periodic orbits is its ability to detect shadowing events even when a turbulent trajectory follows only part of a periodic orbit. As an illustration, in Fig. S4 we show three-dimensional projections (Fig. S4(a)) of a periodic orbit and a turbulent trajectory segment along with the associated persistence diagrams (Fig. S4(b,c)), a recurrence plot (Fig. S4(d)) and the corresponding shadowing distance time series (Fig. S4(e)). Clearly, the shadowing distance $S^{(17)}(t)$ has a local minimum corresponding to this episode whereas the recurrence plot shows no signal at the period $T=17.3382$ of $\overline{\mathrm{po}}_{17}$. This episode is also visualized in the beginning of our supplementary video.
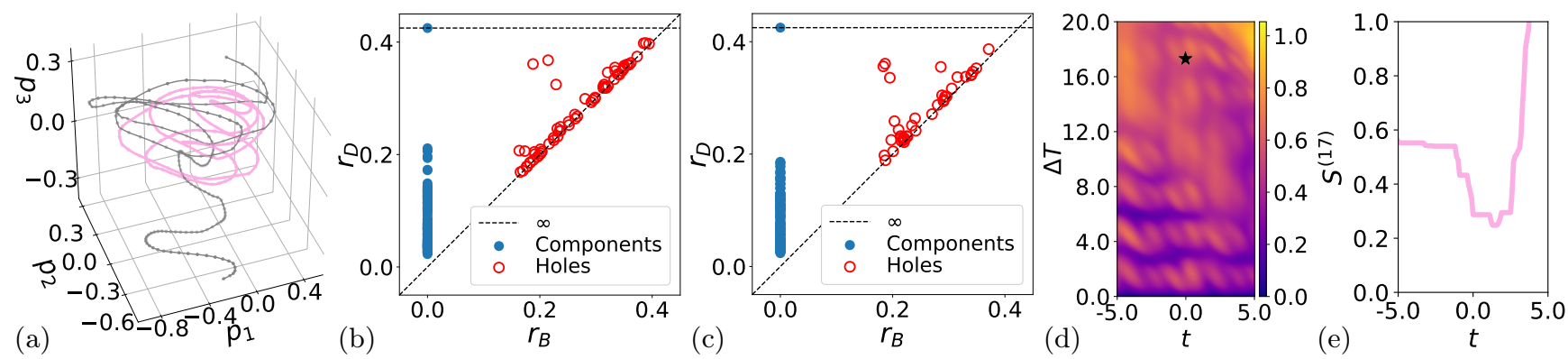

FIG. S4. (a) Periodic orbit $\overline{\mathrm{po}}_{17}$ (pink/thick) and a shadowing trajectory (gray/thin) visualized as projections onto the leading three principal components of $\overline{\mathrm{po}}_{17}$. (b,c) The persistence diagrams associated with $\overline{\mathrm{po}}_{17}$ (b) and shadowing trajectory segment (c) shown in (a). The data points used for generating the persistence diagrams (b,c) are marked with dots along the projection curves in (a). (d) Recurrence plot from a turbulent trajectory, where $t=0$ corresponds to the state in the beginning of the trajectory shown in (a). The marked point (black star) would be the location of the respective recurrence signal, if this state closely recurred to itself after $T_{17}$. (e) Shadowing distance time series corresponding to the same time interval in (a).

\section{ROBUSTNESS AGAINST THE CHOICE OF SHADOWING DISTANCE THRESHOLD}

As explained in the main text, a shadowing event is only registered when the shadowing distance of turbulence from a periodic orbit is less than the threshold $S_{t h}$. Of course, our analysis can only be meaningful if our results do not strongly depend on the choice of this threshold. In Fig. S5 $(\mathrm{a}-\mathrm{c})$ we show cumulative distributions of shadowing distances from $\overline{\mathrm{po}}_{6}, \overline{\mathrm{po}}_{2}$, and $\overline{\mathrm{po}}_{7}$. We chose these as examples in order to illustrate cases corresponding to the highest $\left(\overline{\mathrm{po}}_{6}\right)$ and lowest $\left(\overline{\mathrm{po}}_{7}\right)$ statistical weights and an intermediate one $\left(\overline{\mathrm{po}}_{2}\right)$. Even though the total amount of time that is decomposed into shadowing events varies as a function of our choice of $S_{t h}$, the final invariant distribution changes only slightly for $S_{t h} \in\{0.4,0.5,0.6\}$ as can be seen in Fig. S5(d).
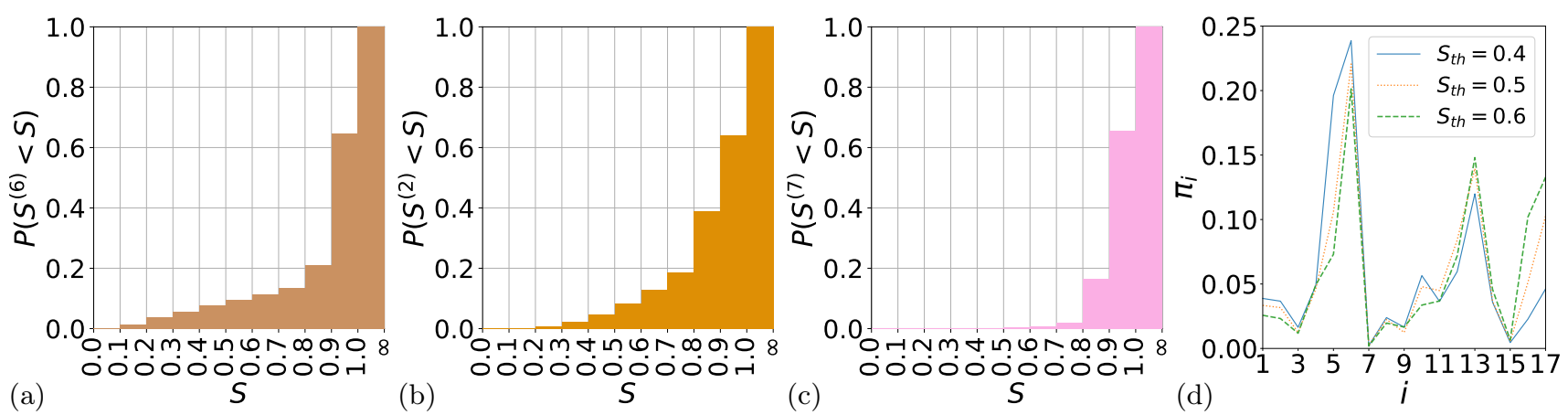

FIG. S5. Cumulative distributions of the shadowing distance of turbulence from (a) $\overline{\mathrm{po}}_{6}$, (b) $\overline{\mathrm{po}}_{2}$, and (c) $\overline{\mathrm{po}}_{7}$. (d) Invariant distribution $(\pi)$ computed using different shadowing distance thresholds $S_{\mathrm{th}}$, with total run time $t_{\mathrm{tot}}=25039$. The data points are connected with line segments for guiding the eye. 


\section{CONVERGENCE OF STATISTICS}

In our analysis, we used three distinct data sets of turbulent dynamics: (i) the recurrence set with the total runtime $t_{\text {tot }}=5864$, (ii) the training set with the total runtime $t_{\text {tot }}=25039$, and (iii) the test set with the total runtime $t_{\mathrm{tot}}=35492$. The sole purpose of the test set was to compute the temporal averages of the kinetic energy and dissipation, convergence of which is shown in Fig. S6. As shown, both averages remain within $1 \%$ of their final value when more than $20 \%$ of the data set is included.

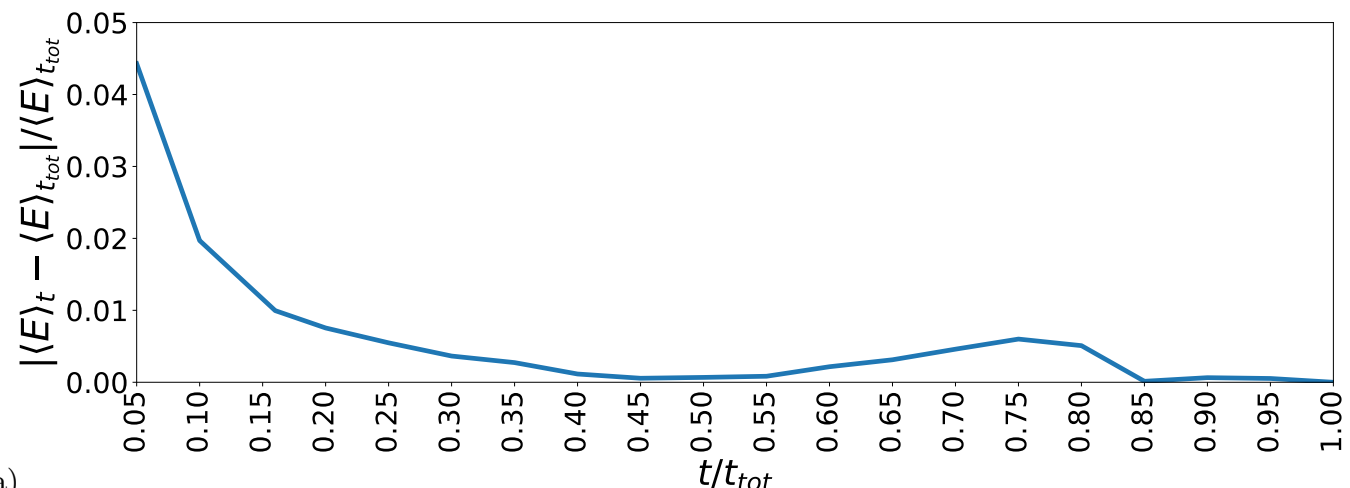

(a)

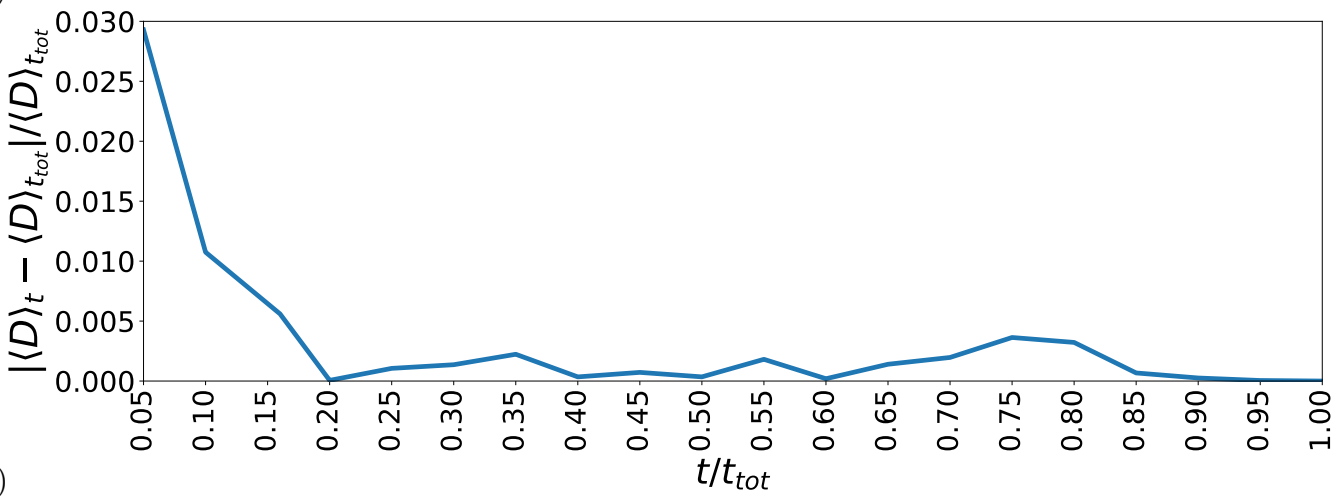

FIG. S6. Relative errors of temporal averages of the (a) kinetic energy and (b) dissipation, computed over the test dataset with a total lifetime $t_{\text {tot }}=19214$.

We use the training data set for the shadowing decomposition of turbulence and, thus, inference of the transition matrix $P$. In Fig. S7(a), we visualized $P$ which we computed using data sets of varying total lengths. In Fig. S7(a), the horizontal axis corresponds to the matrix entries ordered as pairs

$$
[(1,1),(1,2), \ldots(1,17),(2,1),(2,2), \ldots(2,17), \ldots,(17,1),(17,2), \ldots(17,17)] .
$$

In Fig. S7(b), we show the invariant distributions associated with each of these matrices. While Fig. S7(a) appears to be mostly stable, a large peak at $i, j=7,8$ can be seen for $t_{\text {tot }}=25039$, which is not present in the previous estimates. This large fluctuation is a consequence of the fact that $\overline{\mathrm{po}}_{7}$ is not visited often by the turbulent flow, which can also be seen in the probability distribution of $S^{(7)}$ in Fig. S5(c). No such large fluctuation is visible in the invariant distributions shown in Fig. S7(b), suggesting that the long-time behavior inferred from the Markov chain is robust. 
(a)

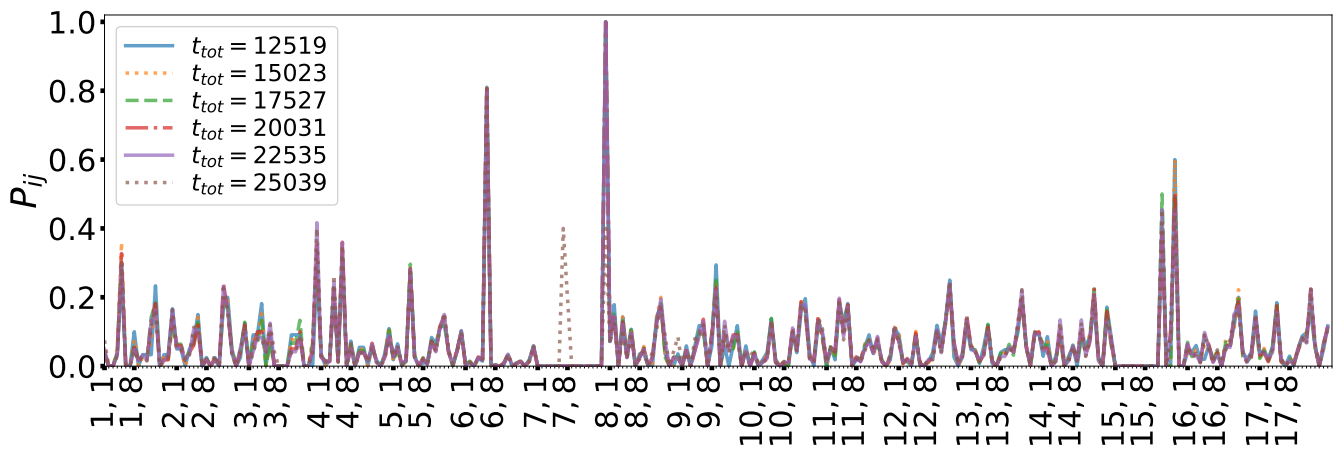

(b)

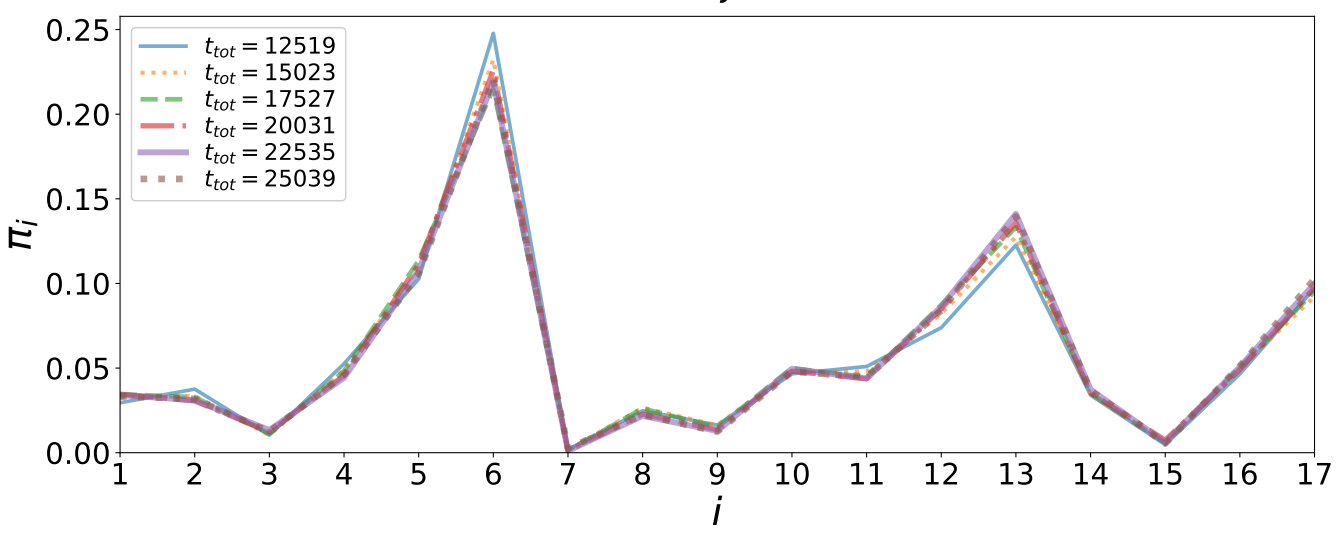

FIG. S7. (a) Transition matrix $(P)$ at different total run times $\left(t_{\text {tot }}\right)$, with $S_{\text {th }}=0.5$. (b) Invariant distribution $(\pi)$ at different total run times $\left(t_{\mathrm{tot}}\right)$, with $S_{\mathrm{th}}=0.5$. The data points are connected with line segments for guiding the eye.

As another illustration of the convergence of our model, we show in Fig. S8(a) the deviation of $P^{t}$ inferred from part of the training data set with duration $t$ from its final estimate $P^{t_{\text {tot }}}$ as measured by the metric

$$
d\left(P^{t}, P^{t_{\mathrm{tot}}}\right)=\sum_{i j} \pi_{i}^{t_{\mathrm{tot}}}\left|P_{i j}^{t}-P_{i j}^{t_{\mathrm{tot}}}\right|,
$$

where the sum is over all matrix entries. In (S6), weighing each row with its contribution to the final invariant measure emphasizes the node contributions accordingly, as well as sets $d\left(0, P^{t_{\mathrm{tot}}}\right)=1$ where 0 is the zero matrix. Additionally, in Fig. S8(b), we show the convergence of the invariant distribution $\pi^{t}$ to its final estimate $\pi^{t_{\mathrm{tot}}}$ using the metric

$$
d\left(\pi^{t}, \pi^{t_{\mathrm{tot}}}\right)=\sum_{i}\left|\pi_{i}^{t}-\pi_{i}^{t_{\mathrm{tot}}}\right| .
$$

Finally, in Fig. S8(c,d), we illustrate the convergence of the mean kinetic energy and dissipation, respectively, as measured by their relative error from the final estimates. 


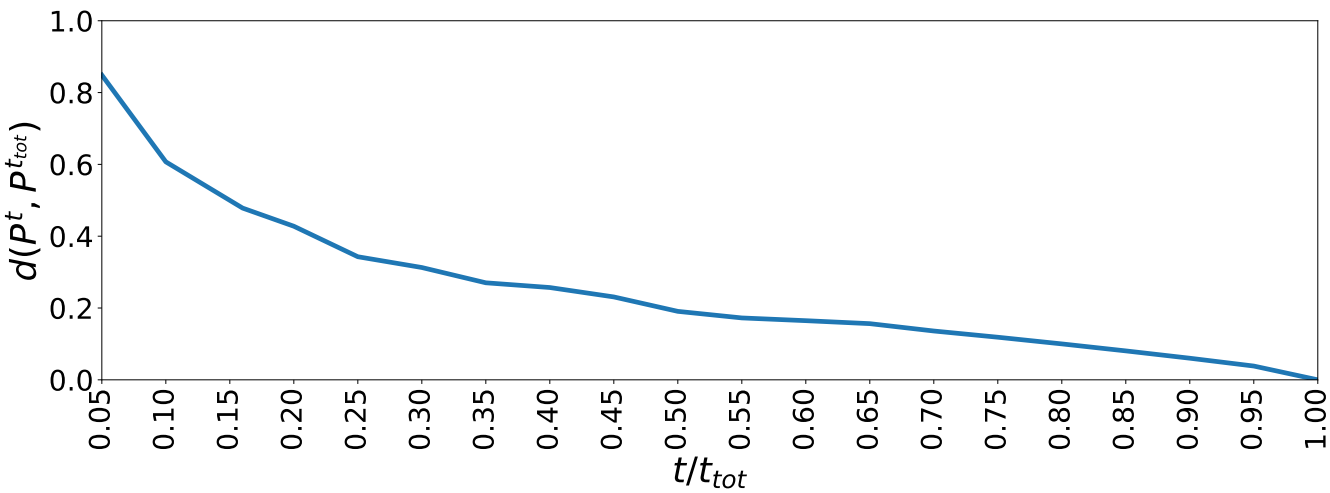

(a)

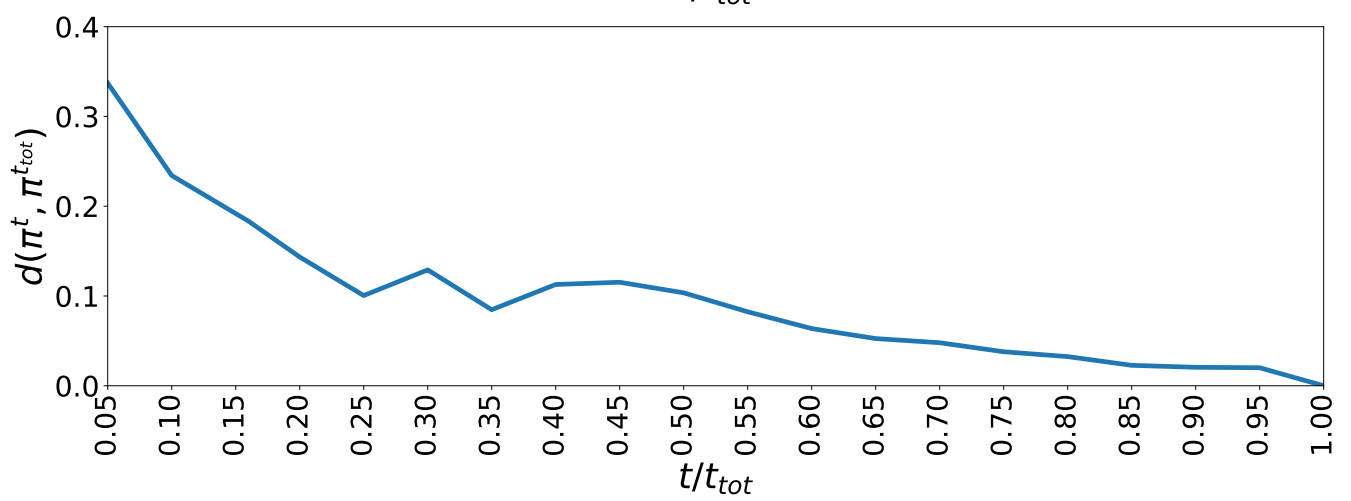

(b)

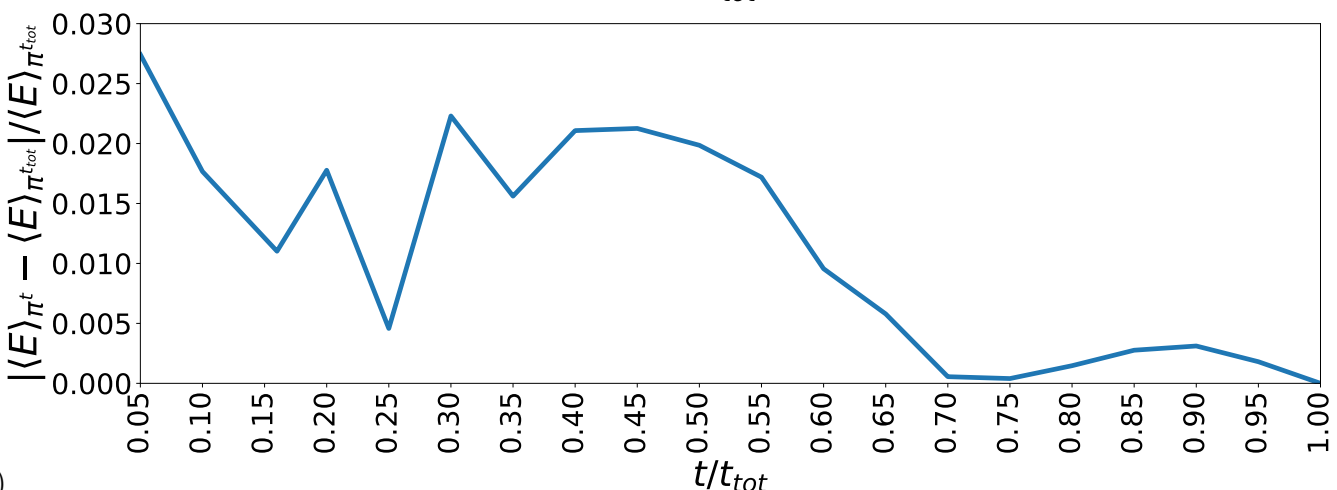

(c)

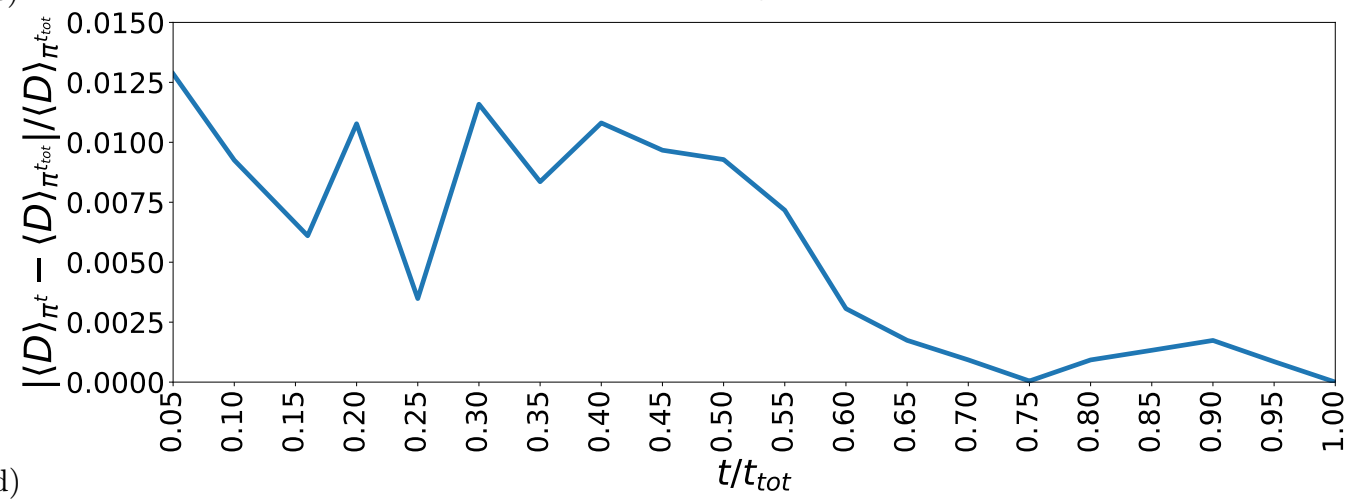

FIG. S8. (a) Convergence of the transition matrix as measured by (S6) as the duration $t$ of training data set is increased. (b) Convergence of the invariant distribution as measured by (S7) as the duration $t$ of training data set is increased. (c,d) Convergence of the mean kinetic energy (c) and dissipation (d) estimates over the invariant distributions $\pi^{t}$. 